\title{
Bone morphogenetic protein 2 inhibits the proliferation and growth of human colorectal cancer cells
}

\author{
YUNYUAN ZHANG ${ }^{1}$, XIAN CHEN $^{1,2,3}$, MIN QIAO $^{1,2}$, BING-QIANG ZHANG $^{1,2}$, NING WANG $^{2,4}$, \\ ZHONGLIN ZHANG ${ }^{2,5}$, ZHAN LIAO $^{2,6}$, LIYI ZENG ${ }^{2,6}$, YOULIN DENG $^{1,2}$, FANG DENG $^{2,4}$, JUNHUI ZHANG ${ }^{1,2}$, \\ LIANGJUN YIN ${ }^{1,2}$, WEI LIU ${ }^{1,2}$, QIAN ZHANG ${ }^{1,2}$, ZHENGJIAN YAN ${ }^{1,2}$, JIXING YE $^{2,7}$, ZHONGLIANG WANG ${ }^{1,2}$, \\ LAN ZHOU $^{1}$, HUE H. LUU ${ }^{2}$, REX C. HAYDON ${ }^{2}$, TONG-CHUAN HE ${ }^{1}$ and HONGYU ZHANG ${ }^{1}$ \\ ${ }^{1}$ Ministry of Education Key Laboratory of Clinical Diagnostic Medicine and the Affiliated Hospitals of Chongqing Medical \\ University, Chongqing 400016, P.R. China; ${ }^{2}$ Molecular Oncology Laboratory, Department of Orthopaedic Surgery, \\ The University of Chicago Medical Center, Chicago, IL 60637, USA; ${ }^{3}$ Department of Laboratory Medicine, \\ The Affiliated Hospitals of Qingdao University, Qingdao 266003; ${ }^{4}$ Departments of Cell Biology and Oncology, \\ Affiliated Southwest Hospital, Third Military Medical University, Chongqing 400038; ${ }^{5}$ Department of Surgery, \\ Affiliated Zhongnan Hospital of Wuhan University, Wuhan 430071; ${ }^{6}$ Departments of Orthopaedic Surgery and \\ General Surgery, The Affiliated Xiang-Ya Hospital of Central South University, Changsha 410008; \\ ${ }^{7}$ School of Bioengineering, Chongqing University, Chongqing 400044, P.R. China
}

Received April 15, 2014; Accepted June 6, 2014

DOI: $10.3892 /$ or.2014.3308

\begin{abstract}
Colorectal cancer (CRC) is one of the most deadly cancers worldwide. Significant progress has been made in understanding the molecular pathogenesis of CRC, which has led to successful early diagnosis, surgical intervention and combination chemotherapy. However, limited therapeutic options are available for metastatic and/or drug-resistant CRC. While the aberrantly activated $\mathrm{Wnt} / \beta$-catenin pathway plays a critical initiating role in CRC development, disruption of the bone morphogenetic protein (BMP) pathway causes juvenile polyposis syndrome, suggesting that BMP signaling may play a role in CRC development. However, conflicting results have been reported concerning the possible roles of BMP signaling in sporadic colon cancer. Here, we investigated the effect of BMP2 on the proliferation, migration, invasiveness and tumor growth capability of human CRC cells. Using an adenovirus vector that overexpresses BMP2 and the piggyBac transposon-mediated stable BMP2 overexpression CRC line,
\end{abstract}

Correspondence to: Dr Tong-Chuan He, Ministry of Education Key Laboratory of Clinical Diagnostic Medicine and School of Clinical Diagnostic Medicine, Chongqing Medical University, Chongqing 400016, P.R. China

E-mail: tche@uchicago.edu

Dr Hongyu Zhang, Department of Surgery, The First Affiliated Hospital of Chongqing Medical University, Chongqing 400016, P.R. China

E-mail: zhy86@hotmail.com

Key words: colorectal cancer, BMP2, BMP signaling, tumorigenesis, proliferation, intestinal epithelial cells we found that exogenous BMP2 effectively inhibited HCT116 cell proliferation and colony formation. BMP2 was shown to suppress colon cancer cell migration and invasiveness. Under a low serum culture condition, forced expression of BMP2 induced a significantly increased level of apoptosis in HCT116 cells. Using a xenograft tumor model, we found that forced expression of BMP2 in HCT116 cells suppressed tumor growth, accompanied by decreased cell proliferation activity. Taken together, our results strongly suggest that BMP2 plays an important inhibitory role in governing the proliferation and aggressive features of human CRC cells.

\section{Introduction}

Colorectal cancer (CRC) causes an average of 50,000 deaths per year in the US and has emerged as the second leading cause of cancer-related mortality in the US and worldwide $(1,2)$. While early diagnosis and surgical intervention, along with combination chemotherapy, has led to improved outcomes, few effective strategies have emerged with which to treat colon cancer once first-line approaches have been exhausted $(1,2)$. The initiation and progression of CRC development are characterized by the accumulation of growing numbers of genetic and epigenetic changes $(3,4)$, while the aberrant activation of the Wnt pathway, either by inactivation of tumor-suppressor adenomatous polyposis coli (APC) or oncogenic activation of $\beta$-catenin, has been demonstrated as the essential initial step of tumorigenesis $(3,5)$. Nonetheless, other alternative pathways have been implicated in CRC development. One involves the formation of serrated adenomas that are associated with mutations in BRAF (6). Another alternative pathway involves the formation of a hamartoma as a precursor lesion, which is in this last rare pathway to CRC that mutations in the bone morphogenetic protein (BMP) pathway were identified (7). 
BMPs belong to the transforming growth factor $\beta$ (TGF $\beta$ ) superfamily (8). BMPs bind to a heterodimeric complex of transmembrane serine threonine kinase receptors type 1 and 2, triggering the phosphorylation and activation of the type 1 receptor by the type 2 receptor kinase. The activated type 1 receptor phosphorylates a receptor-associated SMAD which subsequently complexes with SMAD4 and translocates to the nucleus to regulate gene transcription (9). The importance of BMP signaling in colon cancer development has been highlighted by the identification of mutations in the BMP pathway in colorectal carcinogenesis (7). SMAD4 was identified as being frequently deleted in CRC, although the biological significance of this genetic change has always been attributed to loss of TGF $\beta$ signaling rather than BMP signaling (10). Mutations in BMP receptor 1A (BMPRIA) were found in patients with juvenile polyposis (JP), a rare autosomal dominant hamartomatous polyposis syndrome with an increased risk for the development of CRC (11). Mutations in SMAD4 and BMPRIA account for approximately half of all cases of JP (12-14). Moreover, forced expression of the BMP antagonist noggin in the mouse intestine results in the formation of intestinal hamartomatous polyps (15).

However, conflicting results have been reported concerning the possible roles of BMPs in sporadic colon cancer. For example, several BMPs were found to be growth suppressive and may have their promoters methylated in colon cancer, compatible with a tumor-suppressor role for BMPs in CRC (16-18). However, the expression of BMP4 and BMP7 was found to increase with progression through the adenoma-carcinoma sequence and to correlate with a worse prognosis $(19,20)$. A more recent report showed that BMP signaling promotes the growth of primary human colon cancer in vivo (21). Therefore, the biological effects of BMPs on colon cancer development and progression remain to be fully elucidated.

In the present study, we investigated the effect of BMP2 on the proliferation, migration, invasiveness and tumor growth capabilities of human colon cancer cells. To achieve high levels of exogenous BMP2 expression, we constructed an adenovirus vector that overexpresses BMP2 and also generated the piggyBac transposon-mediated stable BMP2 overexpression cell line using the commonly used human colon cancer line HCT116. We found that exogenous BMP2 effectively inhibited HCT116 cell proliferation and colony formation. BMP2 was shown to suppress colon cancer cell migration and invasiveness as assessed by cell wound healing assay and Boyden chamber Transwell assay. Under a low serum condition, forced expression of BMP2 induced a significantly higher percentage of apoptosis in HCT116 cells than that in the controls. Using a xenograft tumor model, we found that forced expression of BMP2 in HCT116 cells suppressed tumor growth, accompanied by decreased proliferative activity. Thus, our results strongly suggest that BMP2 may play an important inhibitory role in controlling the proliferation and aggressive features of colon cancer cells.

\section{Materials and methods}

Cell culture and chemicals. Human colon cancer cell lines HCT116 and HEK-293 were obtained from the American Type Culture Collection (ATCC; Manassas, VA, USA). The cells were maintained in complete DMEM containing $10 \%$ fetal bovine serum (FBS; Hyclone, Logan, UT), 100 units of penicillin and $100 \mu \mathrm{g}$ of streptomycin at $37^{\circ} \mathrm{C}$ in $5 \% \mathrm{CO}_{2}$ as previously reported (22-27). Unless otherwise indicated, all chemicals were purchased from Sigma-Aldrich (St. Louis, MO, USA) or Thermo Fisher (Pittsburgh, PA, USA).

Recombinant adenoviral vectors expressing BMP2 or GFP. Recombinant adenoviruses were generated using AdEasy technology (28-32). Briefly, the coding regions of human BMP2 and green fluorescent protein (GFP) were PCR amplified and cloned into adenoviral shuttle vectors, which were subsequently used to generate recombinant adenoviruses in HEK-293 cells as previously described $(29,32)$. The resultant recombinant adenoviruses were designated as AdGFP and AdBMP2, respectively. The amplified adenoviruses were titrated and stored at $-80^{\circ} \mathrm{C}$.

Establishment of BMP2/FLuc and FLuc expression stable cell lines. In order to construct BMP2 and/or firefly luciferase (FLuc) stable expression cell lines, the coding regions of human BMP2 and/or FLuc were PCR amplified and subcloned into a homemade piggyBac vector pMPB5, resulting in pMPB-BMP2/FLuc and pMPB-FLuc, respectively. The PCR amplified sequences were verified by DNA sequencing. To construct stable cell lines, exponentially growing HCT116 cells were co-transfected with pMPB-BMP2/FLuc or pMPB-FLuc and the Super piggyBac transposase expression vector (System Biosciences, Mountain View, CA, USA) using Lipofectamine transfection reagents by following the manufacturer's instructions (Life Technologies, Grand Island, NY, USA). At $24 \mathrm{~h}$ after transfection, stable clones were selected in the presence of blasticidin $\mathrm{S}(10 \mu \mathrm{g} / \mathrm{ml})$ for 5 days. The resultant stable cell lines were designated as HCT116-BMP2/FLuc and HCT116-FLuc, respectively. The stable cell lines were verified by RT-PCR for BMP2 expression and/or firefly luciferase activity assay.

Colony formation assay. Exponentially growing HCT116 cells were seeded in 6-well plates at a low density (300 cells/well) and infected with AdGFP or AdBMP2 (MOI=20) for 2 weeks to form colonies. The medium was replaced every 3-4 days. The uninfected cells were also included as a control. The colonies were stained with crystal violet. Each assay condition was conducted in triplicate and repeated in at least three batches of independent experiments. The average colony number for each group was calculated and expressed as the colony formation rate (colony number/seeded cell number) x $100 \%$.

Cell proliferation (MTT) assay. In order to assess cell proliferation and viability, the MTT [3-(4,5-dimethylthiazol-2-yl)-2,5-diphenyltetrazolium bromide] assay was performed as previously described (33-39). Briefly, subconfluent HCT116 cells were infected with AdBMP2 or AdGFP (MOI=20) for $16 \mathrm{~h}$ and seeded in 96 -well plates (1,000 cells/well). The plated cells were incubated in DMEM supplied with $1 \%$ FBS. At the indicated time points, the cells were incubated with $10 \mu 1$ of the CellTiter $96^{\circledR}$ Non-Radioactive Cell Proliferation Assay (MTT) reagent (Promega, Madison, WI, USA) at $37^{\circ} \mathrm{C}$ for $4 \mathrm{~h}$, followed by addition of $100 \mu \mathrm{l}$ DMSO to dissolve the formazan products for $10 \mathrm{~min}$ at room temperature with gentle agitation. The absorbance was measured at $492 \mathrm{~nm}$ using a 
microtiter plate reader. Each assay condition was carried out in five replicates. The overall experiments were repeated at least in three batches of independent experiments.

Cell migration/wound healing assay. Subconfluent HCT116 cells were infected with AdGFP or AdBMP2 for $16 \mathrm{~h}$ and reseeded in 6 -well plates at $\sim 90 \%$ confluency. Upon cell attachment, scratches were made with pipette micro-tips. Floating cells were removed and the attached cells were maintained in DMEM supplemented with $1 \%$ FBS. The width of the scratched cell gaps were monitored and recorded at different time points. The scratch assay was carried out in triplicate and at least three scratch sites were monitored and recorded in each well. Percentage of the wound area closure was measured using ImageJ software.

Boyden chamber invasion/migration assays. The Matrigel cell invasion assay was performed as previously described $(33,34,40)$. Briefly, subconfluent HCT116 cells were infected with AdGFP or AdBMP2 for $24 \mathrm{~h}$. Polycarbonate membranes with $8-\mu \mathrm{m}$ pores were coated with Matrigel (BD Biosciences). The membranes were rehydrated, and $5 \times 10^{5}$ of the transduced cells were placed onto each upper chamber of the Transwell unit. Medium with $10 \%$ FBS was used as a chemoattractant in the bottom chamber. The cells were allowed to invade at $37^{\circ} \mathrm{C}$ in $5 \% \mathrm{CO}_{2}$ for $24 \mathrm{~h}$. Cells were fixed in $10 \%$ formalin and washed with PBS. The cells were stained with hematoxylin and rinsed with water. Cells on the unmigrated side were gently wiped off with a wet cotton tip applicator, and the membrane was rinsed with water. The membranes containing the migrated cells were dried and mounted onto slides with Permount. The number of migrated cells per high power field (HPF) was determined by averaging 20 randomly counted HPFs. The assays were performed in triplicate and repeated in at least three batches of independent experiments.

Apoptosis and flow cytometric analysis. Subconfluent HCT116-FLuc and HCT116-BMP2/FLuc cells were cultured in DMEM containing 1\% FBS for $72 \mathrm{~h}$. Both floating and attached cells were collected, stained with Annexin V-FITC and propidium iodide (PI) using the Annexin V-FITC apoptosis detection kit (BD Pharmingen ${ }^{\mathrm{TM}}$, BD Biosciences). The stained cells were subjected to FACS analysis using the BD $^{\text {TM }}$ LSR II flow cytometer and FlowJo software. Each assay was performed in triplicate and repeated at least three times.

Xenograft tumor growth and xenogen whole body bioluminescence imaging. All animal experiments reported in this study were carried out in strict accordance with the recommendations established in the Guide for the Care and Use of Laboratory Animals of the National Institutes of Health. The protocol was approved by the Institutional Animal Care and Use Committee (IACUC). For subcutaneous xenograft tumor formation, 4-6 week old male athymic nude (nu/nu) mice were purchased from Harlan Sprague Dawley (Indianapolis, IN, USA). Exponentially growing HCT116-BMP2/FLuc and HCT116-FLuc cells were harvested and resuspended in PBS. Cells $\left(2 \times 10^{6}\right.$ in $100 \mu \mathrm{l}$ of PBS) were injected subcutaneously into the flanks of athymic mice ( $n=6 /$ group). All animals were sacrificed 4 weeks after injection.
For weekly whole body bioluminescence imaging, the animals were anesthetized with isoflurane attached to a nose-cone mask within the Xenogen IVIS 200 imaging system. Mice were injected with D-Luciferin sodium salt (Gold BioTechnology, St. Louis, MO, USA) at $100 \mathrm{mg} / \mathrm{kg}$ in $0.1 \mathrm{ml}$ sterile PBS. The pseudo-images were obtained by superimposing the emitted light over the grayscale images of the animal. The average signals in photons $/ \mathrm{sec} / \mathrm{cm}^{2} / \mathrm{stera}$ dian were calculated. Quantitative analysis was carried out using the Xenogen's Living Image software as previously described $(22,35,38,41,42)$.

Histologic evaluation and immunohistochemical staining. The retrieved tissues were fixed in $10 \%$ buffered formalin and embedded in paraffin. The $5-\mu \mathrm{m}$ sections were subjected to H\&E staining. Immunohistochemistry was carried out as previously described $(22,23,30,31,40,43-46)$. For immunohistochemical staining, sections were deparaffinized, rehydrated, subjected to antigen retrieval and probed with a PNCA antibody (Santa Cruz Biotechnology), followed by incubation with biotin-secondary antibodies and streptavidin-HRP. PCNA protein was visualized by 3,3'-diaminobenzidine staining. Control IgG and minus-primary antibody staining were used as negative controls.

Statistical analysis. All quantitative data were calculated and are expressed as means \pm standard deviation. The differences between groups were analyzed using one-way ANOVA followed by the Student-Newman-Keuls test using GraphPad Prism software. A P $<0.05$ was considered to indicate a statistically significant result.

\section{Results and Discussion}

Exogenous BMP2 inhibits the proliferative activity of human colon cancer cells. As the effects of BMP2 on colon cancer cells remain to be fully understood, we used an adenoviral vector overexpressing BMP2 and investigated its effects on the cell proliferation of colon cancer HCT116 cells. Using MTT assay, we found that AdBMP2-infected HCT116 cells exhibited lower proliferative activity at all tested time points when compared with that of the AdGFP-transduced cells, although only the differences on days 3 and 4 exhibited statistical significance $(\mathrm{P}<0.001)$ (Fig. $1 \mathrm{~A})$. When the AdBMP2, AdGFP or uninfected HCT116 cells were seeded at a very low density and allowed to form colonies, the BMP2-expressing HCT116 group formed significantly fewer colonies (Fig. 1B). Quantitatively, the BMP2-expressing HCT116 group formed approximately one third of the number of colonies when compared with the number in the GFP or uninfected control groups (Fig. 1C). These results suggest that BMP2 inhibits the proliferative activity of human colon cancer cells.

BMP2 inhibits the cell migration capability and invasiveness of human colon cancer cells. We further examined whether BMP2 affects the migration capability and invasiveness of colon cancer cells. We performed the commonly used cell wound healing assay to assess the effect on cell migration. The AdBMP2-transduced HCT116 cells were shown to close the scratched gaps on monolayer culture at a much slower pace than 


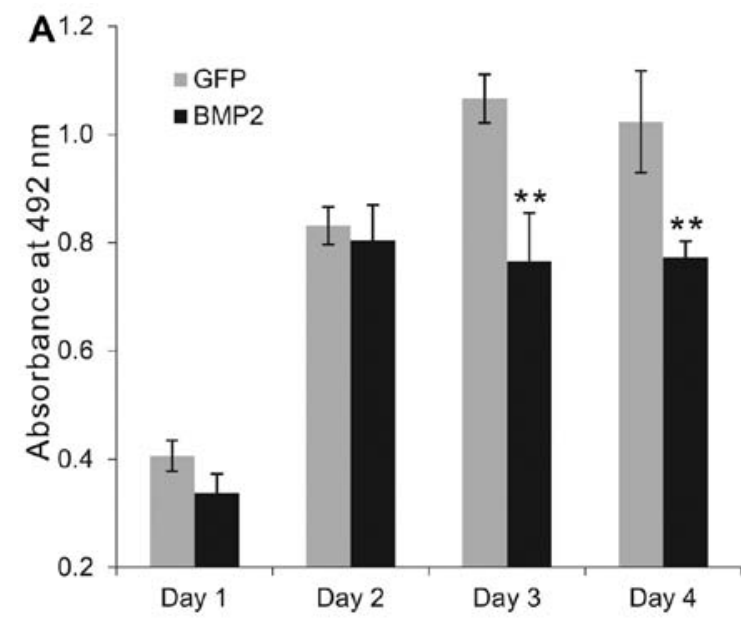

B

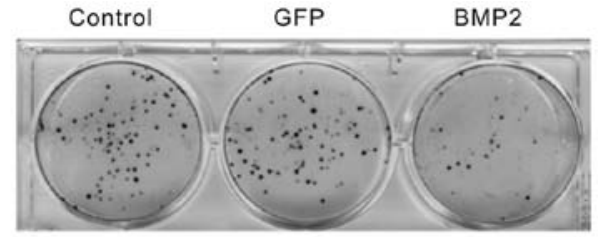

C

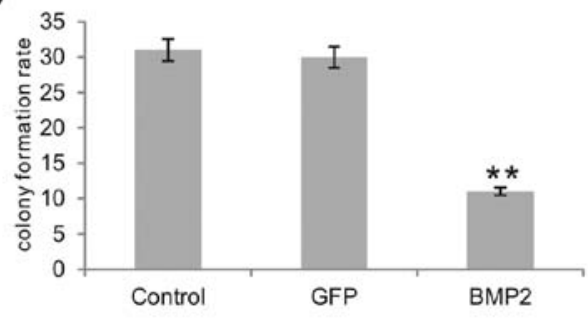

Figure 1. BMP2 inhibits the proliferation and colony formation capability of human colon cancer cells. (A) MTT assay. HCT116 cells were infected with AdGFP or AdBMP2 (MOI=20), seeded in 96-well plates and cultured in DMEM containing 1\% FBS for 4 days. The cells were subjected to MTT assay by measuring the absorbance at $492 \mathrm{~nm}$ using a microplate reader. Each assay condition was carried out in triplicate. ${ }^{* *} \mathrm{P}<0.001$ (BMP2 vs. GFP control). (B and C) Colony formation assay. Exponentially growing HCT116 cells were infected with AdGFP or AdBMP2 (MOI=20) and seeded at a low cell density for 2 weeks to form colonies. The uninfected cells were also included as a control. The colonies were stained with crystal violet. (B) Representative images are shown. (C) Average colony numbers for each group were calculated and are expressed as colony formation rate (colony number/seeded cell number) $\mathrm{x} 100 \%$. Each assay condition was carried out in triplicate and repeated in at least three batches of independent experiments. ${ }^{* *} \mathrm{P}<0.001$ (BMP2 vs. GFP control).
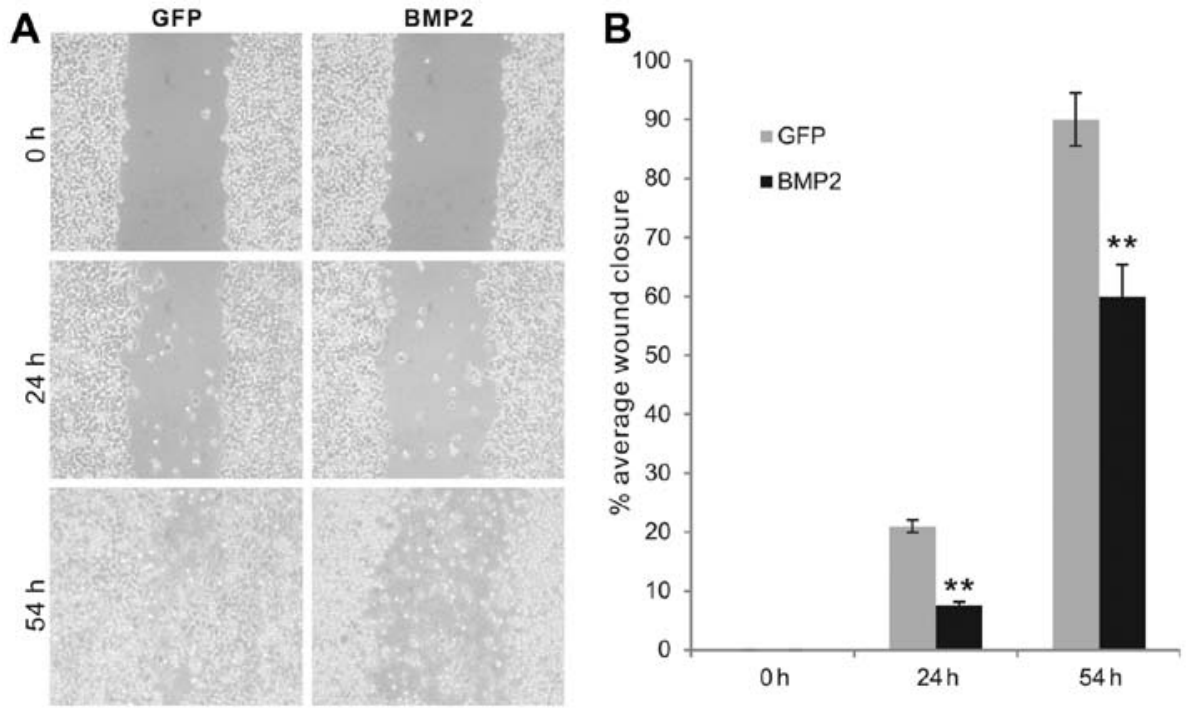

Figure 2. BMP2 inhibits the cell wound healing capability of human colon cancer cells. Subconfluent HCT116 cells were infected with AdGFP or AdBMP2 for $16 \mathrm{~h}$ and reseeded in 6-well plates at $\sim 90 \%$ confluency. Upon cell attachment, scratches were made with pipette micro-tips. Floating cells were removed, and the cells were maintained in DMEM containing 1\% FBS. The width of the scratched cell gaps were monitored and recorded at the indicated time points. The scratch assay was carried out in triplicate and at least three scratch sites were monitored and recorded in each well. (A) Representative images are shown. (B) Percentages of the wound area closure were measured using ImageJ software. Percentages of average wound closure for each group at different time points were graphed. ${ }^{* *} \mathrm{P}<0.001$ (BMP2 vs. GFP).

that of the GFP control groups (Fig. 2A). The percentage of wound closure was significantly higher in the GFP-transduced control cells at all tested time points $(\mathrm{P}<0.001)$ (Fig. 2B). Thus, these results suggest that exogenous BMP2 expression may significantly inhibit the migratory capability of colon cancer cells.

Using the Boyden Transwell extracellular matrix invasion assay, we analyzed the effect of BMP2 on the invasiveness of colon cancer cells. Consistent with our previous reports, GFP-treated HCT116 control cells were fairly aggressive and invaded the Matrigel-coated Transwell membrane with high efficiency, which was inhibited by exogenous BMP2 (Fig. 3A). Quantitatively, the BMP2-transduced HCT116 cells exhibited approximately $<50 \%$ of the number of invaded cells in the GFP control group $(\mathrm{P}<0.001)$ (Fig. 3B), suggesting that BMP2 exerts an inhibitory effect on the invasiveness of colon cancer cells.

BMP2 effectively induces apoptosis in human colon cancer cells. We next investigated whether BMP2 induces apoptosis in colon cancer cells. We established a stable cell line HCT116-BMP2/FLuc that co-expressed human BMP2 

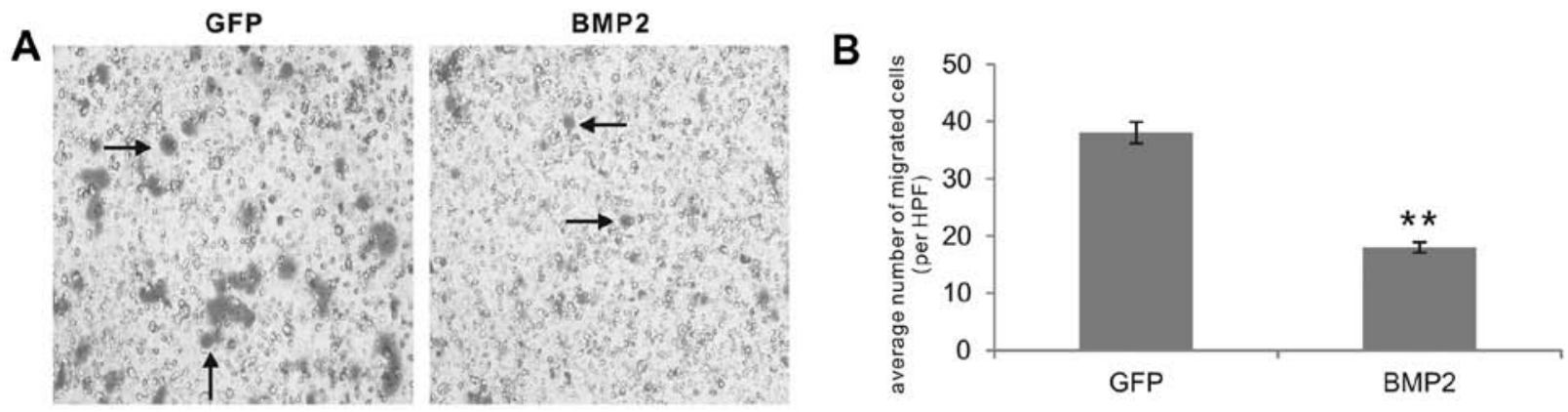

Figure 3. BMP2 inhibits the invasiveness and migratory ability of human colon cancer cells in Boyden chamber invasion/migration assays. Subconfluent HCT116 cells were infected with AdGFP or AdBMP2 for $24 \mathrm{~h}$. The transduced cells were collected and seeded onto 8- $\mu$ m pore Transwell polycarbonate membranes coated with a layer of Matrigel. The cells were allowed to migrate across the membrane using fetal bovine serum as a chemoattractant. Cells that have not migrated across were removed, and the migrated cells were formalin-fixed and stained with H\&E. (A) The numbers of migrated cells were counted under a high power field (HPF, x100) using a microscope. Each assay was carried out in triplicate. The cells migrated across the membrane are indicated by arrows. (B) At least $20 \mathrm{HPFs}$ for each group were counted and the average numbers of the migrated cells were calculated and graphed. ${ }^{* * *} \mathrm{P}<0.001$ (BMP2 vs. GFP).
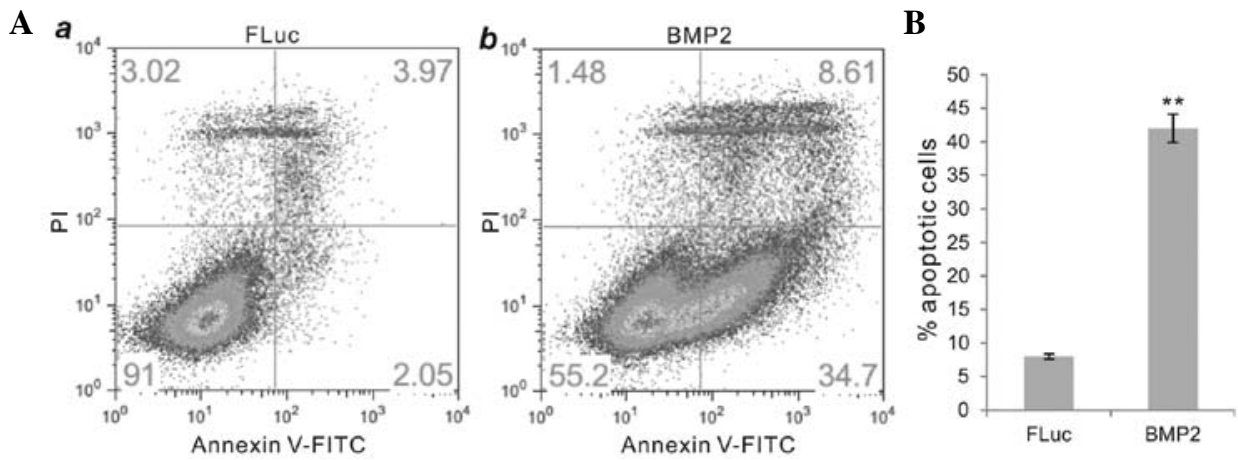

Figure 4. BMP2 effectively induces apoptosis in human colon cancer cells. (A) Subconfluent HCT116-FLuc (a) and HCT116-BMP2/FLuc (b) cells were cultured in DMEM containing 1\% FBS for $72 \mathrm{~h}$. The cells were collected, stained with Annexin V-FITC and propidium iodide (PI) and subjected to FACS analysis. Each assay was carried out in triplicate. (B) Percentages of apoptotic cells were calculated and graphed. ${ }^{* * *} \mathrm{P}<0.001$ (BMP2 vs. FLuc control).

and firefly luciferase (FLuc), while a control stable cell line HCT116-FLuc that only expresses FLuc was established in the same fashion. The exogenous expression of BMP2 was verified by RT-PCR analysis while the FLuc activity was determined using luciferase assay kits. We observed that HCT116-BMP2/FLuc cells grew normally in complete DMEM (with 10\% FBS), compared with the parental HCT116 or HCT116-FLuc cells (data not shown). However, when the BMP2-expressing HCT116 cells were grown under no (0\%) or low (1\%) FBS condition, a significant increase in apoptosis was detected using the Annexin V labeling assay (Fig. 4A, panel a vs. b). When cultured in 1\% FBS/DMEM for $72 \mathrm{~h}$, the BMP2-expressing HCT116 cells underwent significant apoptosis $(\sim 42 \%)$, compared to $\sim 8 \%$ in the control group $(\mathrm{P}<0.001)$ (Fig. 4B). Thus, these results suggest that BMP2 inhibits colon cancer cell proliferation at least in part through induction of apoptosis.

BMP2 effectively inhibits the growth of xenograft tumors derived from human colon cancer. Although the above in vitro data strongly suggest that BMP2 exhibits an inhibitory effect on colon cancer cell proliferation, we aimed to verify whether the inhibitory effect could be extended to in vivo tumor models. We previously demonstrated that HCT116 cells can reproducibly form subcutaneous tumors in athymic nude mice $(35,36)$.
We used the FLuc-tagged stable lines, HCT116-FLuc and HCT116-BMP2/FLuc. Subconfluent HCT116-FLuc and HCT116-BMP2/FLuc (BMP2) cells were subcutaneously injected into athymic nude mice, and the tumor growth was monitored at weeks 2 and 4 using whole body xenogen bioluminescence. We found that the BMP2-expressing HCT116 group formed significantly smaller tumor masses at each time point (Fig. 5A). Quantitative analysis revealed that the tumor growth in the BMP2-expressing HCT116 tumors was $26 \%$ when compared with the control group $(\mathrm{P}<0.03)$ (Fig. 5B). When the retrieved tumor samples were fixed, embedded and sectioned for H\&E staining, the samples from the BMP2/FLuc group exhibited significant necrosis and low cell proliferation, compared with these parameters in the FLuc control group (Fig. 5C, panel a vs. b). The embedded samples were also sectioned and subjected to immunohistochemical staining with a PCNA antibody. We found that the tumor samples formed by HCT116 cells expressing BMP2 exhibited a significantly diminished staining of PCNA expression in the tumor cells (Fig. 5C, panel c vs. d), suggesting that the BMP2expressing colon cancer cells have a decreased proliferative activity. Collectively, these in vivo results further confirm that BMP2 exhibits strong inhibitory effects on colon cancer cells, possibly through inhibiting their proliferation and migration and inducing apoptosis. 

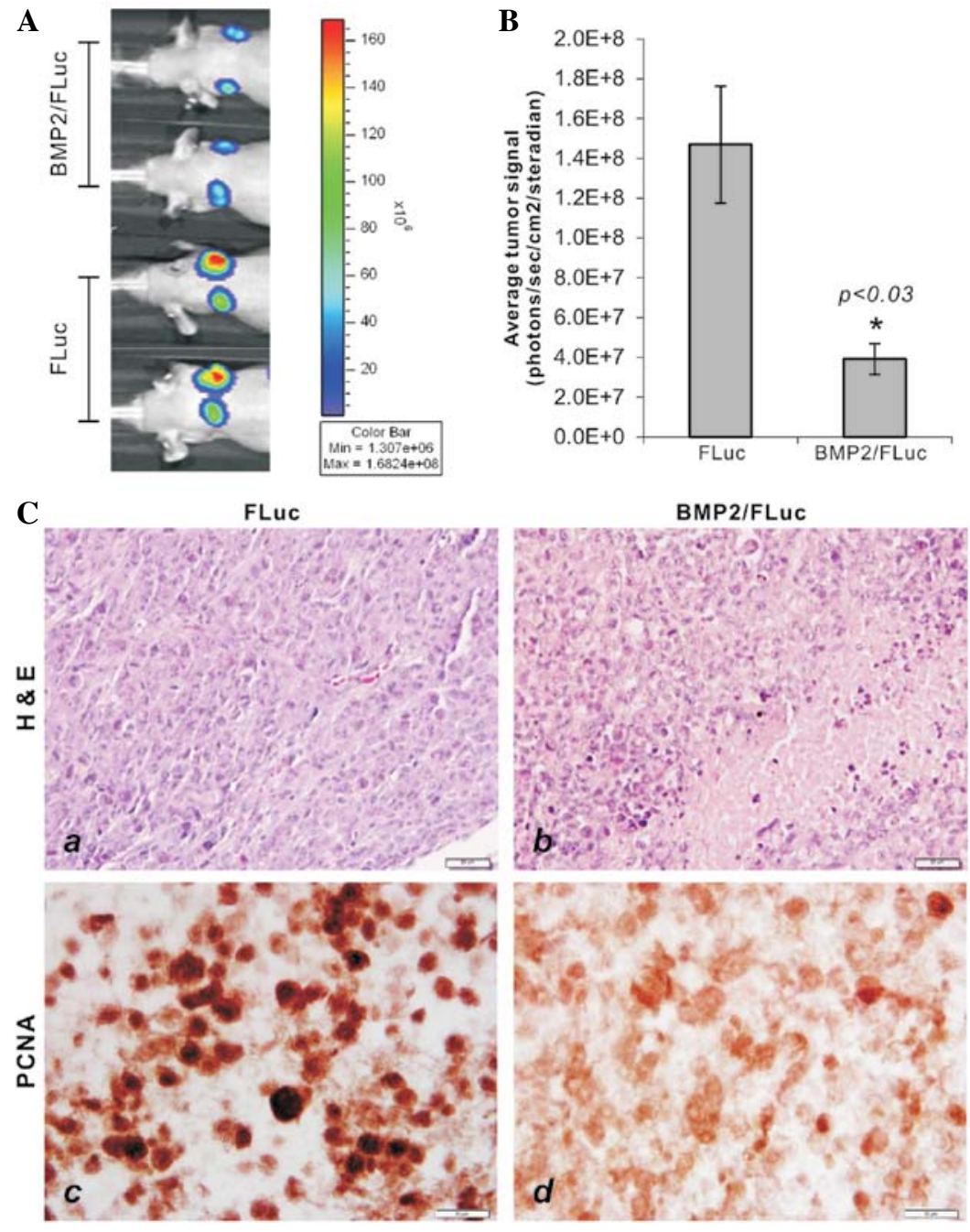

Figure 5. BMP2 inhibits tumor growth in a xenograft model of human colon cancer. (A and B) Xenograft tumor model of human colon cancer. Subconfluent HCT116-FLuc (FLuc) and HCT116-BMP2/FLuc (BMP2/FLuc) cells were collected, resuspended in PBS and subcutaneously injected into athymic nude mice ( $\mathrm{n}=6 ; 2 \times 10^{6}$ cells/injection). (A) Tumor growth was monitored weekly by whole body bioluminescence using the Xenogen IVIS 200 unit. (B) Quantitative data were obtained and analyzed (at week 4). (C) Histologic evaluation and immunohistochemical staining of the proliferation marker PCNA. The retrieved xenograft tumor samples were fixed, paraffin-embedded and sectioned for $\mathrm{H} \& \mathrm{E}$ staining (a and b, scale bar, $20 \mu \mathrm{m}$ ) and immunohistochemical staining with a PCNA antibody (c and d, scale bar, $10 \mu \mathrm{m}$ ). Control IgG and no primary antibody were used as controls (data not shown). Representative results are shown.

BMP signaling may play an important role in modulating colorectal tumorigenesis. The findings from our in vitro and in vivo studies demonstrated that exogenous BMP2 inhibits cell proliferation, migration and invasion, induces apoptosis and suppresses in vivo xenograft tumor growth of human colon cancer cells. The importance of BMP signaling in colorectal tumorigenesis has been highlighted by the identification of frequent mutations of SMAD4 in CRC (10) and mutations in BMP receptor 1A (BMPRIA) in patients with juvenile polyposis (JP) (12-14), which is associated with an increased risk for the development of CRC (11). Moreover, forced expression of the BMP antagonist noggin in mouse intestine was found to result in the formation of intestinal hamartomatous polyps (15).

Consistent with our findings are previous reports in which BMP2, BMP3 or BMP7 were shown to have growth-suppressive activities in colon cancer cells (16-18), although the expression of BMP4 and BMP7 was found to correlate with a worse prognosis $(19,20)$. Notably, a more recent report showed that BMP signaling promotes the growth of primary human colon cancer in vivo and the investigators proposed that blockade of BMP signaling may have beneficial effects against at least a subset of advanced colon cancers (21). Nonetheless, studies have revealed that genetic variations in the BMP signaling pathway may be associated with the etiology, survival and/or prognosis of colon and rectal cancer $(18,47-49)$.

Mechanistically, an early study suggested that BMP2 may act as a tumor suppressor promoting apoptosis in mature colonic epithelial cells (16), although it was suggested that BMP may also utilize SMAD4-independent pathways for growth suppression in colon cancers (18). Notably, it was reported that statins, acting as DNMT inhibitors can demethylate the BMP2 promoter, activate BMP signaling, induce differentiation of colon cancer stem cells and reduce their 'stemness' (50). Moreover, BMP-induced growth suppression may be mediated in part by $\mathrm{p} 21^{\mathrm{WAF} 1}$, which is inhibited by RAS/ERK, as in colon cancer cells where BMP-SMAD signaling and growth suppression are facilitated by $\mathrm{p} 21^{\mathrm{WAF} 1}$ but diminished by oncogenic K-RAS (51). It has been reported 
that suppression of the PI3 kinase/Akt pathway may be correlated with the development of BMP2 resistance and invasion in BMP2-induced epithelial-to-mesenchymal transformation (EMT) in colon cancer (52). It has been reported that the antimitogenic effect of proteasome inhibitors on colon cancer cells may require BMP signaling (53).

In summary, we investigated the effect of BMP2 on the proliferation, migration, invasiveness and tumor growth capabilities of human colon cancer cells. We found that exogenous BMP2 effectively inhibited HCT116 cell proliferation and colony formation. BMP2 also suppressed colon cancer cell migration and invasiveness. Forced expression of BMP2 induced significant apoptosis in HCT116 cells. Using an xenograft tumor model, we found that forced expression of BMP2 in HCT116 cells suppressed tumor growth, accompanied by decreased proliferative activity. Collectively, our results strongly suggest that BMP2 plays an inhibitory role in controlling the proliferation and aggressive features associated with colon cancer cells.

\section{Acknowledgements}

The present study was supported in part by research grants from the National Institutes of Health (CA106569, AT004418, AR50142 and AR054381 to T-C.H, R.C.H. and H.H.L.) and the 973 Program of Ministry of Science and Technology (MOST) of China (\#2011CB707900 to T-C.H.). This study was also supported in part by The University of Chicago Core Facility Subsidy grant from the National Center for Advancing Translational Sciences (NCATS) of the National Institutes of Health through grant no. UL1 TR000430.

\section{References}

1. Bertrand FE,Angus CW,Partis WJ and Sigounas G: Developmental pathways in colon cancer: crosstalk between WNT, BMP, Hedgehog and Notch. Cell Cycle 11: 4344-4351, 2012.

2. Siegel R, Ma J, Zou Z and Jemal A: Cancer statistics, 2014. CA Cancer J Clin 64: 9-29, 2014.

3. Kinzler KW and Vogelstein B: Lessons from hereditary colorectal cancer. Cell 87: 159-170, 1996.

4. Vogelstein B, Papadopoulos N, Velculescu VE, Zhou S, Diaz LA Jr and Kinzler KW: Cancer genome landscapes. Science 339: 1546-1558, 2013.

5. Kinzler KW, Nilbert MC, Su LK, et al: Identification of FAP locus genes from chromosome 5q21. Science 253: 661-665, 1991

6. Chan TL, Zhao W, Leung SY and Yuen ST: BRAF and KRAS mutations in colorectal hyperplastic polyps and serrated adenomas. Cancer Res 63: 4878-4881, 2003.

7. Hardwick JC, Kodach LL, Offerhaus GJ and van den Brink GR Bone morphogenetic protein signalling in colorectal cancer. Nat Rev Cancer 8: 806-812, 2008.

8. Massague J: TGF-beta signal transduction. Annu Rev Biochem 67: 753-791, 1998.

9. Massague J: How cells read TGF-beta signals. Nat Rev Mol Cell Biol 1: 169-178, 2000

10. Thiagalingam S, Lengauer C, Leach FS, et al: Evaluation of candidate tumour suppressor genes on chromosome 18 in colorectal cancers. Nat Genet 13: 343-346, 1996

11. Howe JR, Bair JL, Sayed MG, et al: Germline mutations of the gene encoding bone morphogenetic protein receptor $1 \mathrm{~A}$ in juvenile polyposis. Nat Genet 28: 184-187, 2001.

12. Howe JR, Roth S, Ringold JC, et al: Mutations in the SMAD4/DPC4 gene in juvenile polyposis. Science 280: 1086-1088, 1998.

13. Howe JR, Sayed MG, Ahmed AF, et al: The prevalence of $M A D H 4$ and BMPRIA mutations in juvenile polyposis and absence of $B M P R 2, B M P R 1 B$, and $A C V R 1$ mutations. J Med Genet 41: 484-491, 2004.
14. Sayed MG, Ahmed AF, Ringold JR, et al: Germline SMAD4 or BMPRIA mutations and phenotype of juvenile polyposis. Ann Surg Oncol 9: 901-906, 2002.

15. Haramis AP, Begthel H, van den Born M, et al: De novo crypt formation and juvenile polyposis on BMP inhibition in mouse intestine. Science 303: 1684-1686, 2004.

16. Hardwick JC, Van Den Brink GR, Bleuming SA, et al: Bone morphogenetic protein 2 is expressed by, and acts upon, mature epithelial cells in the colon. Gastroenterology 126: 111-121, 2004.

17. Loh K, Chia JA, Greco S, et al: Bone morphogenic protein 3 inactivation is an early and frequent event in colorectal cancer development. Genes Chromosomes Cancer 47: 449-460, 2008.

18. Beck SE, Jung BH, Fiorino A, et al: Bone morphogenetic protein signaling and growth suppression in colon cancer. Am J Physiol Gastrointest Liver Physiol 291: G135-G145, 2006.

19. Deng H, Makizumi R, Ravikumar TS, Dong H, Yang W and Yang WL: Bone morphogenetic protein- 4 is overexpressed in colonic adenocarcinomas and promotes migration and invasion of HCT116 cells. Exp Cell Res 313: 1033-1044, 2007.

20. Motoyama K, Tanaka F, Kosaka Y, et al: Clinical significance of BMP7 in human colorectal cancer. Ann Surg Oncol 15: 1530-1537, 2008

21. Lorente-Trigos A, Varnat F, Melotti A and Ruiz i Altaba A: BMP signaling promotes the growth of primary human colon carcinomas in vivo. J Mol Cell Biol 2: 318-332, 2010.

22. Luo X, Chen J, Song WX, et al: Osteogenic BMPs promote tumor growth of human osteosarcomas that harbor differentiation defects. Lab Invest 88: 1264-1277, 2008.

23. Tang N, Song WX, Luo J, et al: BMP-9-induced osteogenic differentiation of mesenchymal progenitors requires functional canonical Wnt/beta-catenin signaling. J Cell Mol Med 13: 2448-2464, 2009.

24. Gao Y,Huang E,Zhang H, et al: Crosstalk between Wnt/ $\beta$-catenin and estrogen receptor signaling synergistically promotes osteogenic differentiation of mesenchymal progenitor cells. PloS One 8: e82436, 2013.

25. Kong Y, Zhang H, Chen X, et al: Destabilization of heterologous proteins mediated by the GSK3 $\beta$ phosphorylation domain of the $\beta$-catenin protein. Cell Physiol Biochem 32: 1187-1199, 2013.

26. Zhang $\mathrm{W}$, Zhang $\mathrm{H}$, Wang $\mathrm{N}$, et al: Modulation of $\beta$-catenin signaling by the inhibitors of MAP kinase, tyrosine kinase, and PI3-kinase pathways. Int J Med Sci 10: 1888-1898, 2013.

27. Chen X, Luther G,Zhang W, et al: The E-F hand calcium-binding protein S100A4 regulates the proliferation, survival and differentiation potential of human osteosarcoma Cells. Cell Physiol Biochem 32: 1083-1096, 2013.

28. Cheng H, Jiang W, Phillips FM, et al: Osteogenic activity of the fourteen types of human bone morphogenetic proteins (BMPs). J Bone Joint Surg Am 85-A: 1544-1552, 2003.

29. He TC, Zhou S, da Costa LT, Yu J, Kinzler KW and Vogelstein B: A simplified system for generating recombinant adenoviruses. Proc Natl Acad Sci USA 95: 2509-2514, 1998.

30. Kang Q, Song WX, Luo Q, et al: A comprehensive analysis of the dual roles of BMPs in regulating adipogenic and osteogenic differentiation of mesenchymal progenitor cells. Stem Cells Dev 18: 545-559, 2009.

31. Kang Q, Sun MH, Cheng H, et al: Characterization of the distinct orthotopic bone-forming activity of 14 BMPs using recombinant adenovirus-mediated gene delivery. Gene Ther 11: 1312-1320, 2004.

32. Luo J, Deng ZL, Luo X, et al: A protocol for rapid generation of recombinant adenoviruses using the AdEasy system. Nat Protoc 2: 1236-1247, 2007.

33. Luu HH, Kang Q, Park JK, et al: An orthotopic model of human osteosarcoma growth and spontaneous pulmonary metastasis. Clin Exp Metastasis 22: 319-329, 2005.

34. Luu HH, Zhou L, Haydon RC, et al: Increased expression of S100A6 is associated with decreased metastasis and inhibition of cell migration and anchorage independent growth in human osteosarcoma. Cancer Lett 229: 135-148, 2005

35. He BC, Gao JL, Luo X, et al: Ginsenoside Rg3 inhibits colorectal tumor growth through the down-regulation of Wnt $/ \beta$-catenin signaling. Int J Oncol 38: 437-445, 2011

36. He BC, Gao JL, Zhang BQ, et al: Tetrandrine inhibits Wnt/ $\beta$-catenin signaling and suppresses tumor growth of human colorectal cancer. Mol Pharmacol 79: 211-219, 2011.

37. Su Y, Luo X, He BC, et al: Establishment and characterization of a new highly metastatic human osteosarcoma cell line. Clin Exp Metastasis 26: 599-610, 2009. 
38. Su Y, Wagner ER, Luo Q, et al: Insulin-like growth factor binding protein 5 suppresses tumor growth and metastasis of human osteosarcoma. Oncogene 30: 3907-3917, 2011

39. Wang J, Zhang $\mathrm{H}$, Zhang W, et al: Bone morphogenetic protein-9 (BMP9) effectively induces osteo/odontoblastic differentiation of the reversibly immortalized stem cells of dental apical papilla. Stem Cells Dev: Mar 21, 2014 (Epub ahead of print).

40. Luo Q, Kang Q, Si W, et al: Connective tissue growth factor (CTGF) is regulated by Wnt and bone morphogenetic proteins signaling in osteoblast differentiation of mesenchymal stem cells. J Biol Chem 279: 55958-55968, 2004.

41. Luther GA, Lamplot J, Chen X, et al: IGFBP5 domains exert distinct inhibitory effects on the tumorigenicity and metastasis of human osteosarcoma. Cancer Lett 336: 222-230, 2013.

42. He BC, Chen L, Zuo GW, et al: Synergistic antitumor effect of the activated PPARgamma and retinoid receptors on human osteosarcoma. Clin Cancer Res 16: 2235-2245, 2010.

43. Sharff KA, Song WX, Luo X, et al: Heyl basic helix-loop-helix protein plays an important role in mediating BMP9-induced osteogenic differentiation of mesenchymal progenitor cells J Biol Chem 284: 649-659, 2009.

44. Chen L, Jiang W, Huang J, et al: Insulin-like growth factor 2 (IGF-2) potentiates BMP-9-induced osteogenic differentiation and bone formation. J Bone Miner Res 25: 2447-2459, 2010.

45. Zhang W, Deng ZL, Chen L, et al: Retinoic acids potentiate BMP9-induced osteogenic differentiation of mesenchymal progenitor cells. PloS One 5: e11917, 2010.

46. Luo J, Tang M,Huang J, et al: TGFbeta/BMP type I receptors ALK and ALK2 are essential for BMP9-induced osteogenic signaling in mesenchymal stem cells. J Biol Chem 285: 29588-29598, 2010.
47. Slattery ML, Lundgreen A, Herrick JS, Wolff RK and Caan BJ: Genetic variation in the transforming growth factor- $\beta$ signaling pathway and survival after diagnosis with colon and rectal cancer. Cancer 117: 4175-4183, 2011.

48. Slattery ML, Lundgreen A, Herrick JS, et al: Genetic variation in bone morphogenetic protein and colon and rectal cancer. Int J Cancer 130: 653-664, 2012.

49. Xiang L, Wang S, Jin X, Duan W, Ding X and Zheng C: Expression of BMP2, TLR3, TLR4 and COX2 in colorectal polyps, adenoma and adenocarcinoma. Mol Med Rep 6: 973-976, 2012.

50. Kodach LL, Jacobs RJ, Voorneveld PW, et al: Statins augment the chemosensitivity of colorectal cancer cells inducing epigenetic reprogramming and reducing colorectal cancer cell 'stemness' via the bone morphogenetic protein pathway. Gut 60: 1544-1553, 2011.

51. Beck SE, Jung BH, Del Rosario E, Gomez J and Carethers JM: BMP-induced growth suppression in colon cancer cells is mediated by $\mathrm{p} 21^{\mathrm{WAF} 1}$ stabilization and modulated by RAS/ERK. Cell Signal 19: 1465-1472, 2007.

52. Kang MH, Kang HN, Kim JL, Kim JS, Oh SC and Yoo YA: Inhibition of PI3 kinase/Akt pathway is required for BMP2-induced EMT and invasion. Oncol Rep 22: 525-534, 2009.

53. Wu WK, Sung JJ, Wu YC, Li ZJ, Yu L and Cho CH: Bone morphogenetic protein signalling is required for the anti-mitogenic effect of the proteasome inhibitor MG-132 on colon cancer cells. Br J Pharmacol 154: 632-638, 2008. 